\title{
CARBON AND HYDROCARBON MOLECULES IN VERY COOL WHITE DWARFS
}

\author{
TURGUT ASLAN AND IRMELA BUES \\ Dr. Remeis Sternwarte \\ Astronomisches Institut der Universität Erlangen-Nürnberg \\ 96049 Bamberg, Germany
}

\begin{abstract}
We report on our investigations in progress on the importance of polyatomic molecules - especially of carbon and hydrocarbon - in the atmospheres of very cool helium-rich white dwarfs. For two sets of abundance ratios, model atmospheres with $T_{\text {eff }}=5000 \mathrm{~K}, \log g=8.0$ have been computed and the dependence of $\mathrm{C}_{3}, \mathrm{C}_{2} \mathrm{H}$ and $\mathrm{C}_{2} \mathrm{H}_{2}$ on abundance variations of $\mathrm{H} / \mathrm{He}, \mathrm{C} / \mathrm{He}, \mathrm{O} / \mathrm{He}$ and $\mathrm{N} / \mathrm{He}$ has been calculated in detail, with the absorption of carbon molecules included quantitatively. We discuss the possibility that $\mathrm{C}_{2} \mathrm{H}$ features can explain the observed IR flux deficiency for LHS 1126 at $1.6 \mu \mathrm{m}$ and $2.0-2.3 \mu \mathrm{m}$.
\end{abstract}

\section{Introduction}

At the very cool end of the white dwarf cooling sequence with $T_{\text {eff }} \leq 5500 \mathrm{~K}$, the objects are intrinsically faint. Even if they belong to the solar neighborhood within $25 \mathrm{pc}$, they cannot be observed with high resolution. Spectra in the blue show only weak features, and some have a pure continuum.

Even observations with a high signal/noise ratio allow only a classification (Greenstein \& Liebert 1990). Strong line features of Ca (Ca I $\lambda 4227 \AA$, $\mathrm{Ca}$ II $\mathrm{H} \& \mathrm{~K}$ ) and Fe can be seen in LHS 69 and strong band features of $\mathrm{CH}$ and $\mathrm{C}_{2}$ in G99-37, thus indicating helium-rich compositions with and without carbon.

New model atmospheres for G99-37 $\left(T_{\text {eff }}=6000 \mathrm{~K}, \log g=8.0, \mathrm{He} / \mathrm{H}\right.$ $=1000, \mathrm{C} / \mathrm{H}=35.5)$ with $\mathrm{C}_{3}$ included have shown that one feature in the blue can be attributed to this molecule (Bues \& Karl-Dietze 1995). G99-37 has a magnetic field of $2 \times 10^{3}$ Tesla which might be responsible for the intermediate ratio of $\mathrm{H} / \mathrm{He}$ compared to other white dwarfs with carbon features in their spectra. The former conclusion that cooler objects with 
shifted $\mathrm{C}_{2}$ bands have the same ratios of $\mathrm{C} / \mathrm{He}$ and the shifts of the features are due to a magnetic field is no longer valid for all observed objects, according to the polarization measurements by Schmidt et al. (1995), which showed no polarization at all for ESO 439-162 and LHS 1126. These authors suggest the possibiliy that the observed features are not due to $\mathrm{C}_{2}$ at all but belong to polyatomic molecules. Our current investigation is aimed at very cool helium-rich white dwarfs where the flux in the infrared region must be affected severely by the presence of molecular features, even for reduced abundances and the corresponding increase of gas pressure, which could cause shifts in wavelength of the same order as a magnetic field.

\section{Model Atmospheres}

New flux-constant model atmospheres have been calculated for extremely helium-rich compositions in the range $5500 \mathrm{~K}>T_{\text {eff }}>4800 \mathrm{~K}, \log g=8.0$, with varied relative abundances of $\mathrm{H} / \mathrm{He}, \mathrm{C} / \mathrm{He}, \mathrm{O} / \mathrm{He}$ and $\mathrm{N} / \mathrm{He}$, where oxygen is most important for gas pressure.

The possible $\mathrm{H}, \mathrm{C}, \mathrm{O}$ reaction processes were critically reviewed. Because of the high gas pressure and a ratio $\mathrm{C} / \mathrm{H} \geq 1$ in agreement with the presence of $\mathrm{C}_{2}$ and $\mathrm{C}_{3}$ features, some formation processes have been preferred compared to those in normal carbon stars, namely:

$$
\begin{aligned}
& \mathrm{C}_{2}+\mathrm{C} \rightarrow \mathrm{C}_{3} \\
& \mathrm{C}_{2}+\mathrm{H} \rightarrow \mathrm{C}_{2} \mathrm{H} \\
& \mathrm{C}_{2}+\mathrm{H}_{2} \rightarrow \mathrm{C}_{2} \mathrm{H}_{2}
\end{aligned}
$$

while other processes for the formation of $\mathrm{C}_{2} \mathrm{H}$ and $\mathrm{C}_{2} \mathrm{H}_{2}$ such as

$$
\begin{aligned}
& \mathrm{C}_{2}+\mathrm{H}_{2} \rightarrow \mathrm{C}_{2} \mathrm{H}+\mathrm{H} \\
& \mathrm{C}_{2} \mathrm{H}+\mathrm{H} \rightarrow \mathrm{C}_{2} \mathrm{H}_{2} \\
& \mathrm{C}_{2} \mathrm{H}+\mathrm{H}_{2} \rightarrow \mathrm{C}_{2} \mathrm{H}_{2}+\mathrm{H}
\end{aligned}
$$

have been omitted.

The constants of dissociation for $\mathrm{C}_{3}$ and $\mathrm{C}_{2} \mathrm{H}_{2}$ were taken from Tsuji (1964), and for $\mathrm{C}_{2} \mathrm{H}$ from Perić et al. (1990). The computation of dissociative equilibria included all diatomic molecules and neutral and ionized species of $\mathrm{H}, \mathrm{He}, \mathrm{C}, \mathrm{N}$, and $\mathrm{O}$ and was solved with an iterative scheme. For absorption, the molecules were included in a smeared-line approximation, appropriate for white dwarfs due to the high pressure. For $\mathrm{C}_{2} \mathrm{H}$, transitions at $9500 \mathrm{~cm}^{-1}(=1.05 \mu \mathrm{m})$ and $5500 \mathrm{~cm}^{-1}(=1.82 \mu \mathrm{m})$ calculated by Reimers et al. (1985), and transitions at $1.78 \mu \mathrm{m}, 1.81 \mu \mathrm{m}$ and $2.61 \mu \mathrm{m}$ calculated by Perić et al. (1992) with ab initio methods, have been included in our calculations.

Figure 1 shows relative partial pressures of the molecules $\mathrm{H}_{2}, \mathrm{C}_{2}, \mathrm{CN}$, $\mathrm{CO}, \mathrm{CH}, \mathrm{C}_{3}, \mathrm{C}_{2} \mathrm{H}$ and $\mathrm{C}_{2} \mathrm{H}_{2}$ compared to the total gas pressure versus the optical depth for a model atmosphere with the indicated parameters 


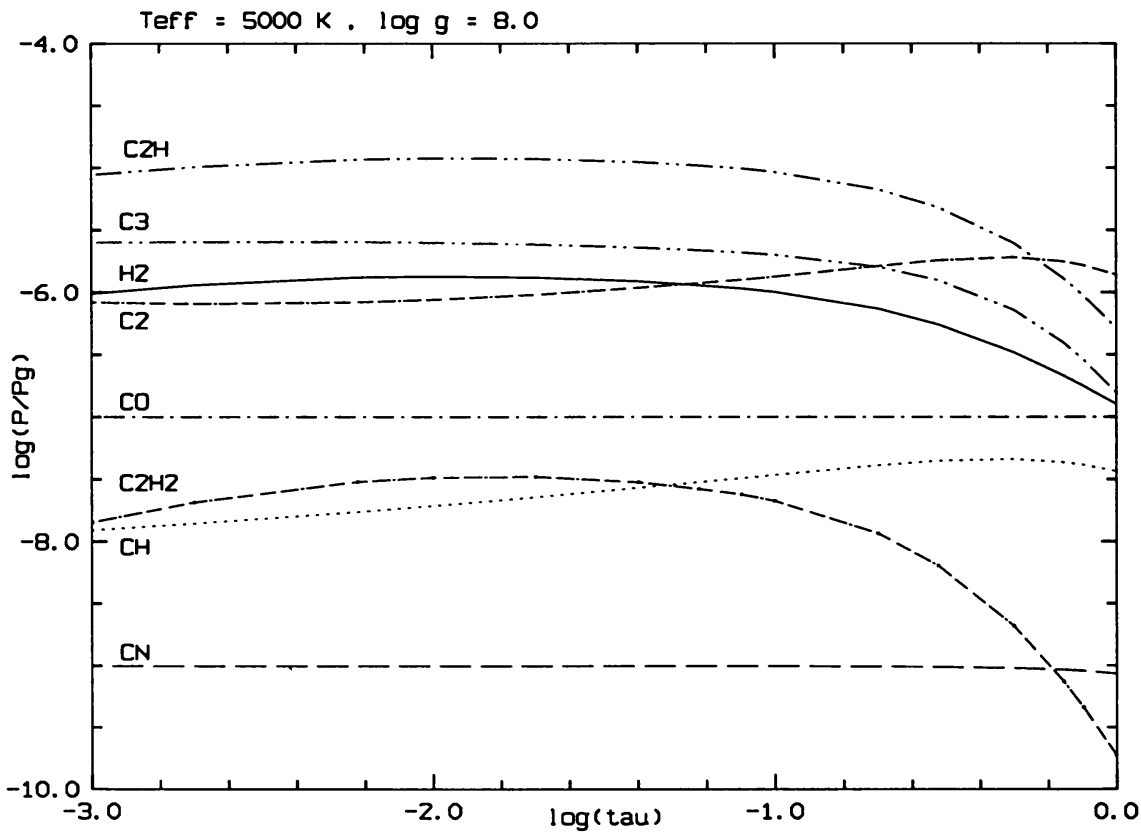

Figure 1. Pressure fractions of various diatomic and polyatomic molecules versus the optical depth for $T_{\text {eff }}=5000 \mathrm{~K}, \log g=8.0, \log (\mathrm{H} / \mathrm{He})=-5, \log (\mathrm{C} / \mathrm{He})=-5, \log (\mathrm{O} / \mathrm{He})$ $=-7$, and $\log (\mathrm{N} / \mathrm{He})=-9$.

relevant for LHS 1126. The optical depth scale in the line-forming region is $\tau$-Rosseland.

Obviously the $\mathrm{C}_{2} \mathrm{H}$ molecule is the most dominant one in the composition given in Fig. 1, followed by $\mathrm{C}_{3}, \mathrm{H}_{2}$ and $\mathrm{C}_{2}$. Diatomic $\mathrm{CO}$ and $\mathrm{CH}$ as well as $\mathrm{C}_{2} \mathrm{H}_{2}$ play important minor roles, while $\mathrm{CN}$ can be neglected.

A further reduction in $\mathrm{H}$ and $\mathrm{C}$ as well as in the relative abundance ratio yields the results shown in Figure 2. Both figures can be compared to Fig. 4 of Schmidt et al. (1995) where, for a fixed value of $P_{g}$, the relative abundances of hydrocarbons, dependent on temperature only, are shown for a mixture of just $\mathrm{H}, \mathrm{He}$ and $\mathrm{C}$. Our model-atmosphere stratification includes a steep gradient of temperature and gas pressure as well, where the $\mathrm{O}$ abundance is responsible for the formation of polyatomic molecules due to the formation of $\mathrm{CO}$ and the corresponding high pressure.

In Fig. 2 this effect is well demonstrated. Although the ratio $\mathrm{C} / \mathrm{H}$ is changed by a factor of $3, \mathrm{CO}$ is still the most abundant molecule; next, with smaller values than for the first mixture, we find $\mathrm{C}_{2}, \mathrm{C}_{3}$ and $\mathrm{C}_{2} \mathrm{H}$. As for depressions in flux caused by observable features, this means that $\mathrm{C}_{2}$ features should be visible, at least at this $T_{\text {eff }}$. The molecules $\mathrm{CN}, \mathrm{H}_{2}$ and 


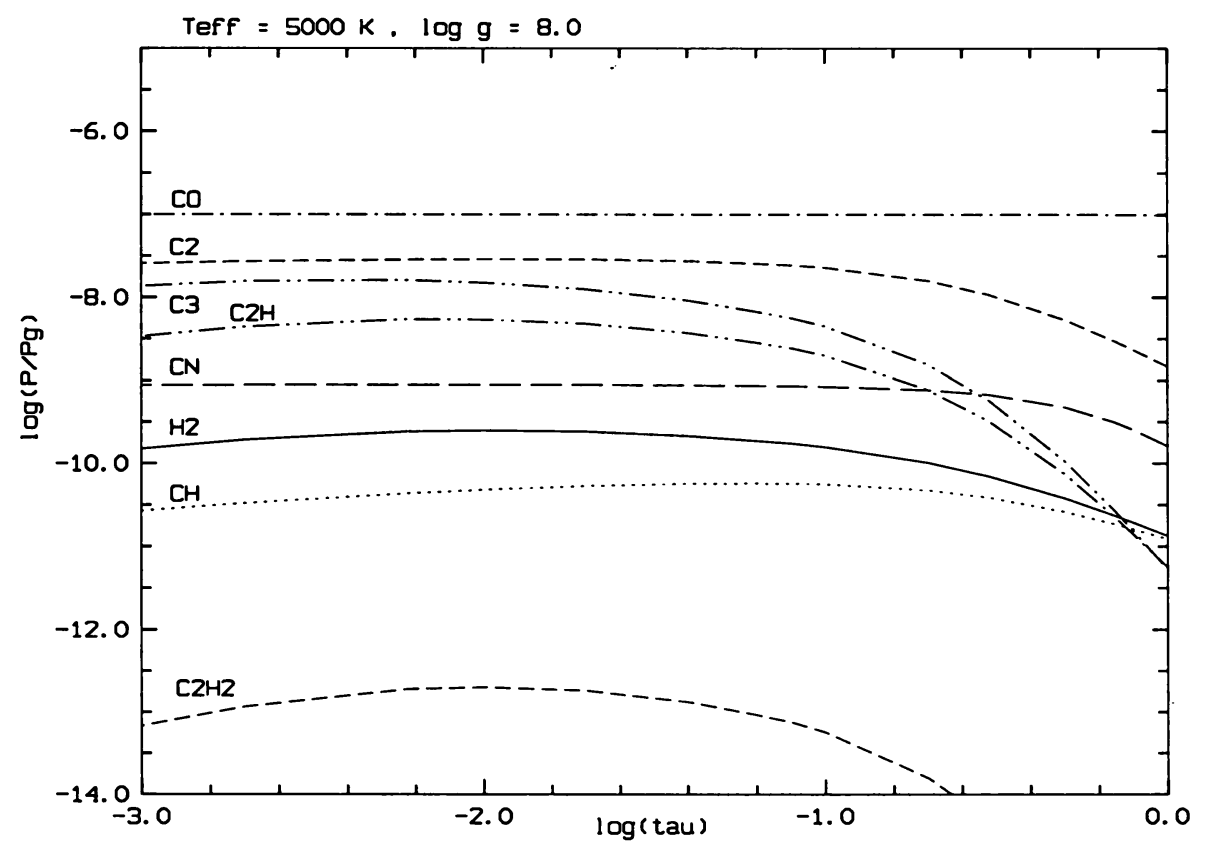

Figure 2. Pressure fractions for reduced abundances: $T_{\text {eff }}=5000 \mathrm{~K}, \log g=8.0$, $\log (\mathrm{H} / \mathrm{He})=-7, \log (\mathrm{C} / \mathrm{He})=-6.5, \log (\mathrm{O} / \mathrm{He})=-7, \log (\mathrm{N} / \mathrm{He})=-9$.

$\mathrm{CH}$ play a minor role, and $\mathrm{C}_{2} \mathrm{H}_{2}$ can be neglected.

\section{LHS 1126}

One object to which these computations can be applied is LHS 1126. The optical spectrum shows a $12 \%$ molecular absorption band at $4990 \AA \pm$ $100 \AA$. Broad absorption bands at $5450 \AA \pm 200 \AA, 6050 \AA \pm 200 \AA$ and $6680 \AA \pm 100 \AA$ are present in addition to $\mathrm{C}_{3} \lambda 4050 \AA$. Our infrared spectrum, taken in May 1995, shows a weak depression near $9200 \AA$.

The star has already been analyzed by Bergeron et al. (1994) with blue and red spectra and model atmospheres consisting of hydrogen and helium only. The observed features at $4590 \AA$ and $4990 \AA$ were attributed to $\mathrm{C}_{2}$, yet the shift in wavelength cannot, in their opinion, be due to pressure effects alone as their ratio $\mathrm{H} / \mathrm{He}=0.8$ gives more electrons than our computations. To account for the infrared flux distribution, which does not correspond to a black body as shown by Lebofsky \& Liebert (1984), they propose collisioninduced absorption by molecular hydrogen due to collisions with helium, yet they do not discuss absorption by $\mathrm{CH}$ or other carbon molecules. They obtain $T_{\text {eff }}=5400 \mathrm{~K} \pm 200 \mathrm{~K}, \log g=7.85 \pm 0.17$, and $\log (\mathrm{He} / \mathrm{H})=0.8$ 


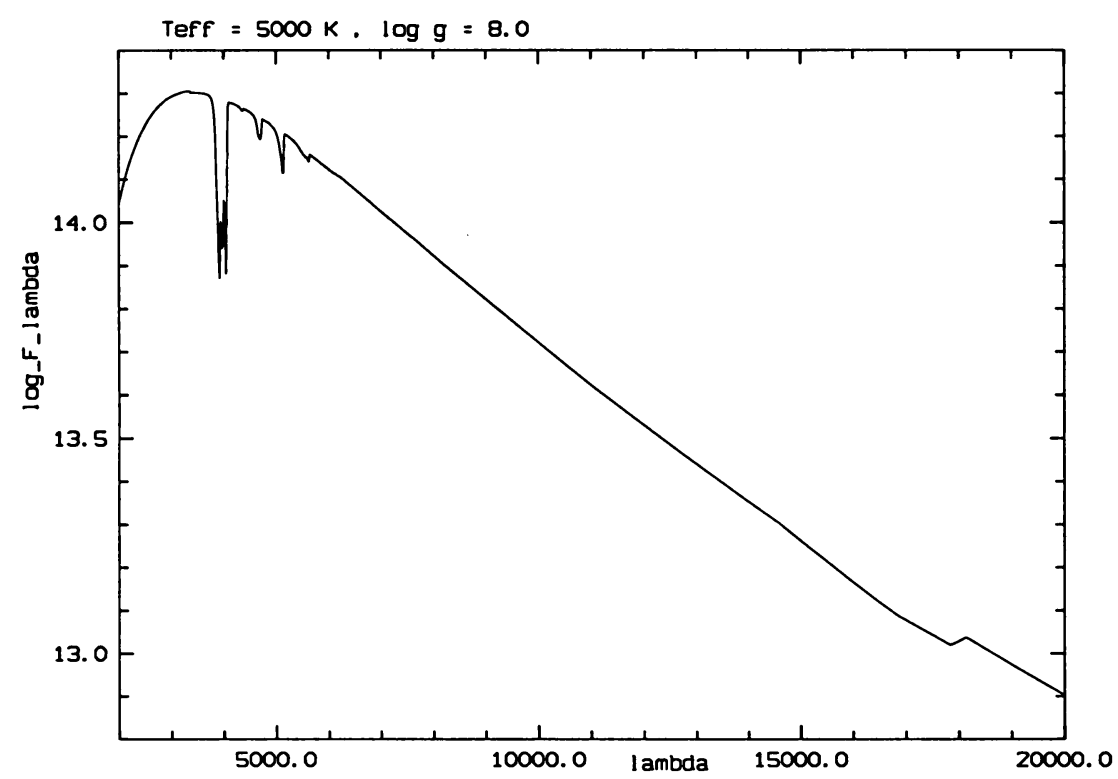

Figure 3. Calculated flux for the parameters given in Fig. 2. In addition to the $\mathrm{C}_{2}$ and $\mathrm{C}_{3}$ bands in the visual, a broad absorption feature at $\lambda=1.78 \mu \mathrm{m}$ is present.

\pm 0.2 for this object.

Schmidt et al. (1995) discussed the observed features in LHS 1126 and concluded that the absence of the G-band of $\mathrm{CH}$ is an indication of a lower hydrogen abundance than the value determined by Bergeron et al. (1994). They investigated the relative abundances of $\mathrm{C}_{n}$ and $\mathrm{C}_{n} \mathrm{H}_{n}$ compounds for a fixed value of gas pressure, and $\mathrm{C} / \mathrm{He}$ as a function of temperature $(\mathrm{H} / \mathrm{He}$ $=0.1$ and $\mathrm{C} / \mathrm{He}=10^{-2}, 10^{-3}$ and $\left.10^{-5}\right) . \mathrm{C}_{2} \mathrm{H}$ is one of their preferably formed molecules in the photosphere of white dwarfs, a result confirmed by our computations.

With $T_{\text {eff }}=5000 \mathrm{~K}, \log g=8.0, \log (\mathrm{H} / \mathrm{He})=-5, \log (\mathrm{C} / \mathrm{He})=-5, \log$ $(\mathrm{O} / \mathrm{He})=-5$, and $\log (\mathrm{N} / \mathrm{He})=-9$ (Aslan et al. 1996), agreement with the observed flux in the visible was possible. Further analysis showed that an even lower relative abundance of carbon and hydrogen was sufficient, and therefore two sets of parameters were used for the calculations (see captions to Figs. 1 and 2).

Information about the infrared region of LHS 1126 has been obtained by photometry with six filters in the $J H K$ region by Lebofsky \& Liebert (1984), and by spectroscopy around $2.0 \mu \mathrm{m}$ by Bergeron et al. (1994). The observed flux deficiency between $1.6 \mu \mathrm{m}$ and $2.1 \mu \mathrm{m}$ could not be matched completely by the model spectra. 
For the abundance ratios of Fig. 2, the corresponding flux of our model atmosphere is shown in Figure 3 from the blue to the infrared region of the spectrum. Features in the blue are mainly due to $\mathrm{C}_{3}$ and $\mathrm{C}_{2}$, and in the infrared to $\mathrm{C}_{2}$ and $\mathrm{C}_{2} \mathrm{H}$. Our results can explain the $1.78 \mu \mathrm{m}$ region but not the $2.1 \mu \mathrm{m}$ feature. The consistency with the observed spectrum in the $1.6 \mu \mathrm{m}$ region is better than in the computations by Bergeron et al. (1994). Further steps of iteration in the details of abundance ratios and absorption processes in these extremely high-pressure model atmospheres are necessary.

\section{References}

Aslan, T., Bues, I. \& Karl-Dietze, L. 1996, in Hydrogen-Deficient Stars, ed. C. S. Jeffery and U. Heber, ASP Conf. Ser., 96, 325

Bergeron, P., Ruiz, M.-T., Leggett, S. K., Saumon, D. \& Wesemael, F. 1994, ApJ, 423, 456

Bues, I. \& Karl-Dietze, L. 1995, in White Dwarfs, ed. D. Koester and K. Werner (SpringerVerlag), Lecture Notes in Physics, No. 443, p. 201

Greenstein, J. L. \& Liebert, J. W. 1990, $A p J, 360,662$

Lebofsky, M. J. \& Liebert, J. W. 1984, $A p J, 278$, L111

Perić, M., Peyerimhoff, S. D. \& Buenker, R. J. 1990, Mol. Phys., 71, 693

Perić, M., Peyerimhoff, S. D. \& Buenker, R. J. 1992, Z. Phys. D, 24, 177

Reimers, J.R., Wilson, K. R., Heller, E.J. \& Langhoff, S. R. 1985, J. Chem. Phys., 82, 5064

Schmidt, G. D., Bergeron, P. \& Fegley, B. Jr. 1995, ApJ, 443, 274

Tsuji, T. 1964, Ann. Tokyo Astron. Obs., 2nd ser., 9, 1

\section{Discussion}

Jørgensen: Is $\mathrm{C}_{2} \mathrm{H}$ stable under the conditions of your models? Where did you take your data for the $\mathrm{C}_{2} \mathrm{H}$ molecule from?

Aslan: Our calculations show that $\mathrm{C}_{2} \mathrm{H}$ is stable and is responsible for the IR flux deficiency at $1.6 \mu \mathrm{m}$ ( $H$ band). There is a strong transition at 1.78 $\mu \mathrm{m}$. We took the data for $\mathrm{C}_{2} \mathrm{H}$ from an article by Perić et al. (1992).

Bues: These data are $a b$ initio calculations. 